\title{
Aplicación del proceso Fenton en la disminución de materia orgánica en aguas residuales de la industria termoeléctrica
}

\author{
MIGUEL TACO UGSHA', EDUARDO MAYORGA LLERENA ${ }^{\text {* }}$ \\ 'Universidad Central del Ecuador, Facultad de Ciencias Químicas, \\ Ciudadela Universitaria, Quito-Ecuador (teléfono: 00593-2-3215035). \\ * Correspondencia: emayorga@uce.edu.ec
}

Recibido: 29 febrero 2012 / Aceptado: 13 julio 2012

\section{Resumen}

En el presente trabajo se empleó al proceso Fenton para la remoción de materia orgánica en aguas residuales provenientes de la industria termoeléctrica que usa como combustible fuel oil. El proceso de degradación se siguió a través de la medición de la Demanda Química de Oxígeno, (DQO). El trabajo se inició con la caracterización de las aguas residuales con mediciones de $D Q O$, que sirvieron como punto de partida en el estudio de la influencia de las variables de operación, que fueron: el pH (2.5 y 3.0) y la relación de 1/60 a 1/15 de las concentraciones (en $\mathrm{mg} / \mathrm{L}$ ) de los reactivos para la reacción Fenton, $\mathrm{Fe}^{2+} / \mathrm{H}_{2} \mathrm{O}_{2}$., las mismas que no mostraron ser significativas a temperatura ambiente. Los porcentajes de remoción de DQO conseguidos (entre el 72.51 y 88.60\%) permitieron cumplir con la Ordenanza 213 del Distrito Metropolitano de Quito que especifica un máximo de $123 \mathrm{mg} / \mathrm{L}$ de DQO. Además, se estudió la evolución de la remoción de materia orgánica en función del tiempo para una muestra de agua residual que presentó una $D Q O$ inicial de $357.14 \mathrm{mg} \mathrm{O}_{2} / \mathrm{L}$, obteniéndose una reducción significativa de $D Q O$ (del 89.68\%) en un tiempo de reacción de 352 minutos.

Palabras clave: Proceso Fenton, radical hidroxilo, materia orgánica, demanda química de oxígeno, fuel oil.

\section{Fenton process application in decrease of organic matter in thermoelectric industrial wastewaters}

\section{Abstract}

In this paper was used the Fenton process for removal of organic matter in wastewater from the thermoelectric industry that uses fuel oil, the degradation process was followed by measuring the chemical oxygen demand (COD). The work began with the characterization of wastewater COD measurements, which served as a starting point to study the influence of operating variables, these were: $\mathrm{pH}(2.5$ and 3.0) and the ratio of 1/60 to $1 / 15$ of the concentrations (in $\mathrm{mg} / \mathrm{L}$ ) of the reagents for the Fenton reaction, $\mathrm{Fe}^{2+} / \mathrm{H}_{2} \mathrm{O}_{2}$, these are not statistically significant at room temperature, the percentage of COD removal were ranged between 72.51 and $88.60 \%$, these percents were enough for complying with Order 213 of the Metropolitan District of Quito, which specifies a maximum COD of $123 \mathrm{mg} / \mathrm{L}$. In addition, the removal of organic matter evolution was studied with a wastewater sample with $357.14 \mathrm{mgO}_{2} / \mathrm{L}$ of $\mathrm{COD}$, a great reduction of $\mathrm{COD}(89.68 \%)$ was achieved in a reaction time of 352 minutes.

Key words: Fenton process, hydroxyl radical, organic matter, chemistry oxygen demand, fuel oil. 


\section{Introducción}

La generación de energía eléctrica, a partir de la combustión de fuel oil, implica el uso del agua para mantenimiento y limpieza de piezas mecánicas en una central térmica. Las aguas residuales producidas contienen alta carga orgánica formada principalmente por residuos de fueloil, diésel y aceites lubricantes que contienen compuestos tóxicos y peligrosos tales como: hidrocarburos aromáticos policíclicos (PAH) [1], olefinas, alquilbencenos, fenoles, entre otros. Muchos de estos contaminantes son resistentes a la biodegradación y no pueden eliminarse en su totalidad por métodos convencionales [2]. Estas aguas comúnmente son tratadas mediante métodos convencionales físicos, químicos o fisicoquímicos como: separación de residuos de combustible por diferencia de densidad entre agua y contaminante (en piscinas API), cloración, coagulación-floculación y clarificación, lo que genera una disminución en la DQO; sin embargo, estos procedimientos no siempre son suficientes para cumplir con la normativa ambiental dentro del Distrito Metropolitano de Quito (DMQ) cuyo valor es de $123 \mathrm{mg} / \mathrm{L}$. Los procesos de oxidación avanzada, AOPs (Advanced Oxidation Processes), son una alternativa para el tratamiento de efluentes industriales debido a su capacidad de degradación de compuestos orgánicos recalcitrantes [3]. Uno de éstos es el proceso Fenton, que consiste en la reducción del $\mathrm{H}_{2} \mathrm{O}_{2}$ para formar el radical hidroxilo, $\bullet \mathrm{OH}$, catalizada por $\mathrm{Fe}^{2+}$ en medio ácido. Este radical es el segundo agente oxidante más fuerte después del flúor y es capaz reaccionar con una gran variedad de compuestos orgánicos e inorgánicos hasta alcanzar la mineralización de los mismos [4], su potencial rédox es $2.80 \mathrm{~V}$ vs SHE [3]; la reacción para formar el radical hidroxilo es la siguiente (reacción 1):

$$
\mathrm{H}_{2} \mathrm{O}_{2}+\mathrm{Fe}^{2+} \longrightarrow \mathrm{Fe}^{3+}+\cdot \mathrm{OH}+\mathrm{OH}^{-} ; \kappa=76 \mathrm{M}^{-1} \cdot \mathrm{s}^{-1}
$$

El catalizador se regenera por la reacción 2, permitiendo la producción de radicales hidroperóxido $(\bullet \mathrm{OOH})$, los cuales participan en la oxidación a menor velocidad comparado con los $\bullet \mathrm{OH}$.

$$
\mathrm{Fe}^{3+}+\mathrm{H}_{2} \mathrm{O}_{2} \longrightarrow \mathrm{Fe}^{2+}+\cdot \mathrm{OOH}+\mathrm{H}^{+} ; \varkappa=0.01 \mathrm{M}^{-1} \cdot \mathrm{s}^{-1}
$$

La eficiencia de la reacción de Fenton está gobernada principalmente por un control adecuado de las canti- dades de $\mathrm{H}_{2} \mathrm{O}_{2}$, del catalizador, del $\mathrm{pH}$ y de la temperatura [5]. El proceso Fenton es uno de los AOPs más eficientes para el tratamiento de efluentes industriales y se ha empleado para eliminar contaminantes como: pesticidas [6], lixiviados [7], tintes [8], productos farmacéuticos y de cuidado personal (PPCPs) [5], residuos de destilado de vino [9], aceite de maíz [10], entre otros. Entre las ventajas que presenta este método están: la disponibilidad y bajo costo de las sales de hierro, la ausencia de peligro para el medio ambiente de los productos de descomposición del $\mathrm{H}_{2} \mathrm{O}_{2}$ [3] y su instalación no requiere equipos adicionales, como ocurre en el caso de la ozonización o la radiación UV; además, los costos de operación son bajos ya que se lo puede hacer en condiciones de presión y temperatura ambiente.

\section{Parte experimental}

\subsection{Toma de muestras}

La técnica de muestreo empleada fue de tipo compuesto, y consistió en la recolección de muestras reales de aguas residuales de la planta de tratamiento de la Central Térmica ubicada en Guangopolo. Los volúmenes parciales fueron de $200 \mathrm{~mL}$, tomados aproximadamente cada 2 minutos hasta completar un volumen total de $4 \mathrm{~L}$.

\subsection{Métodos analíticos}

La determinación de cloruros $\left(4500-\mathrm{Cl}^{-} \mathrm{B}\right.$, método argentométrico) y DQO (5220 D, método titulométrico a reflujo cerrado) se efectuaron acordes al Standard Methods for the Examination of Water and Wastewater [1 1]; y el pH, se determinó con un medidor de $\mathrm{pH}$ Metler Toledo S2O Seven Easy.

\subsection{Variables}

Se estudió el efecto de los siguientes parámetros:

- Concentración (en $\mathrm{mg} / \mathrm{L}$ ) de los reactivos de Fenton en las proporción $\mathrm{Fe}^{2+} / \mathrm{H}_{2} \mathrm{O}_{2}: 1 / 60,1 / 30,1 / 20$ y $1 / 15$; y

- $\mathrm{pH}: 2.5$ y 3.0 .

\subsection{Procedimiento experimental}

En cada ensayo se aplicó el siguiente procedimiento: se tomaron $400 \mathrm{~mL}$ de agua residual en un vaso de precipitación de $600 \mathrm{~mL}$, se ajustó el pH de las 
muestras con $\mathrm{H}_{2} \mathrm{SO}_{4}$ concentrado hasta 2.5 ó 3.0, de acuerdo al experimento; luego, con agitación, se agregó $\mathrm{FeSO}_{4} \cdot 7 \mathrm{H}_{2} \mathrm{O}$ sólido y $\mathrm{H}_{2} \mathrm{O}_{2} 30 \%$ en cantidades necesarias para lograr las proporciones de $\mathrm{Fe}^{2+} / \mathrm{H}_{2} \mathrm{O}_{2}$. En todos los ensayos se empleó agitación mecánica con velocidad de $200 \mathrm{rpm}$. Después de cuatro horas de reacción, se adicionó un exceso de $\mathrm{FeSO}_{4} \cdot 7 \mathrm{H}_{2} \mathrm{O}$ sólido, hasta conseguir una concentración de 2000 $\mathrm{mg} / \mathrm{L}$ de $\mathrm{Fe}^{2+}$ e inmediatamente se llevó a pH básico agregando gotas de una solución de $\mathrm{KOH} 6 \mathrm{~N}$. Después se sedimentaron y clarificaron las muestras que rápidamente se filtraron empleando papel filtro cualitativo $(\varnothing 90 \mathrm{~mm})$ para su caracterización final.

La cantidad de peróxido de hidrógeno, asignada en cada ensayo, estuvo en función del nivel de contaminación de la muestra. Para la adición de éste, se empleó la siguiente expresión (ecuación 3):

$$
\mathrm{C}_{\mathrm{H}_{2} \mathrm{O}_{2}}=\frac{17}{8} \mathrm{DQO}
$$

Donde $\mathrm{CH}_{2} \mathrm{O}_{2}$ es la concentración de peróxido de hidrógeno en $\mathrm{mg} / \mathrm{L}$. A esta concentración se denomina la "dosis estequiométrica referida a la DQO", la cual es la necesaria para la oxidación de la materia orgánica [3].

\section{Resultados y discusión}

\subsection{Caracterización físico-química de las aguas residuales de partida}

Los resultados de la caracterización físico-química de las aguas residuales en el punto de muestreo, se presentan en la tabla 1.

Tabla 1. Caracterización físico-química del agua residual *

\begin{tabular}{cccc}
\hline Parámetro & Unidad & $\begin{array}{c}\text { Valor } \\
\text { mínimo }\end{array}$ & $\begin{array}{c}\text { Valor } \\
\text { máximo }\end{array}$ \\
\hline DBO5 & $\mathrm{mg} / \mathrm{L}$ & 39.0 & 63.0 \\
DQO & $\mathrm{mg} / \mathrm{L}$ & 132.44 & 357.14 \\
Aceites y grasas & $\mathrm{mg} / \mathrm{L}$ & 17.4 & 23.80 \\
Cloruros & $\mathrm{mg} / \mathrm{L}$ & 242.30 & 250.56 \\
$\mathrm{TPH}$ & $\mathrm{mg} / \mathrm{L}$ & 7.64 & 7.9 \\
$\mathrm{pH}$ & ----- & 5.18 & 10.60 \\
Conductividad & $\mathrm{mS} / \mathrm{cm}$ & 716 & 1218 \\
Turbidez & $\mathrm{NTU}$ & 9.50 & 13.66 \\
\hline *Datos han sido obtenidos en muestras que se tomaron en doce días distintos.
\end{tabular}

\subsection{Demanda química de oxígeno de las muestras tratadas mediante la oxidación Fenton}

Finalizado el proceso de oxidación, en cada muestra y condición empleada, se lograron descensos significativos de DQO. Dichos resultados se muestran en la figura 1. Las líneas entrecortadas representan los resultados luego de emplear un $\mathrm{pH}$ de 3.0; mientras que las continuas, corresponden a los obtenidos a pH de 2.5. Se observa que las líneas entrecortadas se encuentran, en su mayoría, por debajo de las continuas, indicando que al emplear el pH de 3.0, se obtienen niveles de DQO más bajos que a pH 2.5. Para la relación $\mathrm{Fe}^{2+} / \mathrm{H}_{2} \mathrm{O}_{2}$, no se observan tendencias claras a medida que la dosis de catalizador aumenta.
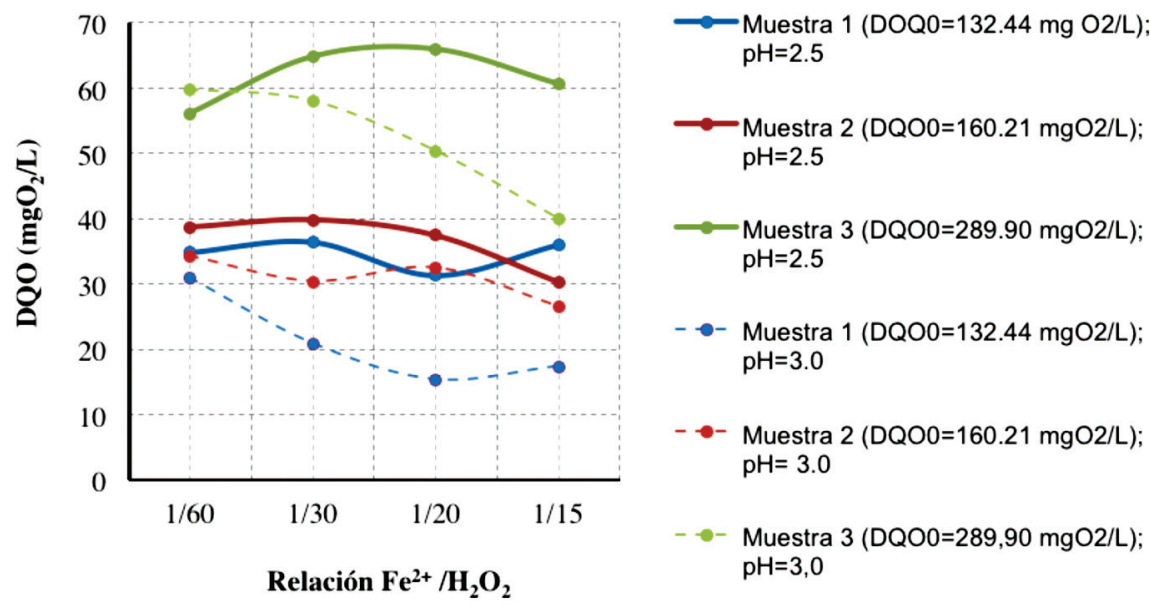

Figura 1. Demanda química de oxígeno, para tres muestras tratadas mediante el proceso Fenton, a diferente $\mathrm{pH}$ y a diferentes relaciones $\mathrm{Fe}^{2+} / \mathrm{H}_{2} \mathrm{O}_{2}$ 
Los valores finales de DQO, en todos los casos, estuvieron por debajo del nivel máximo considerado en la legislación cuyo valor señalado es de $123 \mathrm{mgO}_{2} / \mathrm{L}$. En la tabla 2, se muestran los resultados de la remo- ción de DQO con sus combinaciones experimentales, distribuidos en tres bloques formados por muestras correspondientes a descargas realizadas en tres diferentes días (bloques I, II y III).

Tabla 2. Combinaciones experimentales y porcentajes de remoción (volumen total $=400 \mathrm{~mL}$ )

\begin{tabular}{|c|c|c|c|c|c|c|}
\hline & \multicolumn{2}{|c|}{$\begin{array}{c}\text { Bloque I } \\
\text { (muestra 1) }\end{array}$} & \multicolumn{2}{|c|}{$\begin{array}{c}\text { Bloque II } \\
\text { (muestra 2) }\end{array}$} & \multicolumn{2}{|c|}{$\begin{array}{c}\text { Bloque III } \\
\text { (muestra 3) }\end{array}$} \\
\hline $\begin{array}{l}\text { Relación } \\
\mathrm{mg} \mathrm{Fe}^{2+} / \mathrm{mg} \mathrm{H}_{2} \mathrm{O}_{2}\end{array}$ & 2.5 & 3.0 & 2.5 & 3.0 & 2.5 & 3.0 \\
\hline $1 / 60$ & $73.69 \%$ & $76.67 \%$ & $75.87 \%$ & $76.67 \%$ & $81.26 \%$ & $80.00 \%$ \\
\hline $1 / 30$ & $72.51 \%$ & $84.24 \%$ & $75.13 \%$ & $81.07 \%$ & $78.33 \%$ & $80.59 \%$ \\
\hline $1 / 20$ & $76.36 \%$ & $88.44 \%$ & $76.61 \%$ & $79.77 \%$ & $77.93 \%$ & $83.14 \%$ \\
\hline $1 / 15$ & $72.84 \%$ & $86.93 \%$ & $81.07 \%$ & $83.45 \%$ & $79.71 \%$ & $86.60 \%$ \\
\hline
\end{tabular}

\subsection{Análisis de la relación $\mathrm{Fe}^{2+} / \mathrm{H}_{2} \mathrm{O}_{2}$ y del pH en la remoción de la DQO}

Para el análisis de la influencia de la relación $\mathrm{Fe}^{2+}$ / $\mathrm{H}_{2} \mathrm{O}_{2}$ y del pH en el proceso Fenton, se consideró el diseño experimental de parcelas subdivididas en el cual, cada bloque (muestra) es dividido en dos partes llamadas parcelas completas que corresponden a los valores del $\mathrm{pH}$. Cada parcela completa es dividida en cuatro partes llamadas subparcelas que están asignadas por cada una de las relaciones $\mathrm{Fe}^{2+} / \mathrm{H}_{2} \mathrm{O}_{2}$ [12]. Los resultados del ANOVA, para el diseño de parcelas subdivididas, se indican en la tabla 3 .

Tabla 3. Variaciones y cuadrados medios obtenidos para los resultados en el análisis de varianza para el diseño de parcelas subdivididas.

\begin{tabular}{cccccc}
\hline Fuente de variación & Variación & $\begin{array}{c}\text { Grados de } \\
\text { libertad }\end{array}$ & $\begin{array}{c}\text { Cuadrado } \\
\text { medio }\end{array}$ & $\begin{array}{c}\mathbf{F} \\
\text { calculada }\end{array}$ & $\begin{array}{c}\mathbf{F}_{0.95} \\
\text { tabulada }\end{array}$ \\
\hline Bloques & 21.02 & 2 & 10.51 & & \\
$\mathrm{pH}$ & 194.29 & 1 & 194.29 & 16.42 & 18.51 \\
$\begin{array}{c}\text { Error de la parcela completa } \\
\text { (Bloque } \times \mathrm{pH} \text { ) }\end{array}$ & 23.67 & 2 & 11.83 & & \\
\hline $\begin{array}{c}\mathrm{Fe}^{2+} / \mathrm{H}_{2} \mathrm{O}_{2} \\
\text { Bloque } \times \mathrm{Fe}^{2+} / \mathrm{H}_{2} \mathrm{O}_{2}\end{array}$ & 59.09 & 3 & 19.70 & 2.92 & 5.39 \\
$\mathrm{pH} \times \mathrm{Fe}^{2+} / \mathrm{H}_{2} \mathrm{O}_{2}$ & 40.46 & 6 & 6.74 & & \\
$\begin{array}{c}\text { Error de la subparcela } \\
\left.\text { (bloques } \times \mathrm{pH} \times \mathrm{Fe}{ }^{2+} / \mathrm{H}_{2} \mathrm{O}_{2}\right)\end{array}$ & 36.07 & 3 & 12.02 & 1.17 & 3.00 \\
\hline Total & 61.61 & 6 & 10.27 & & \\
\hline
\end{tabular}

Los resultados de la tabla 3 indican que los valores obtenidos de $\mathrm{F}$, para: el pH, la relación $\mathrm{Fe}^{2+} / \mathrm{H}_{2} \mathrm{O}_{2}$ y para la interacción entre ambas variables, son menores que sus correspondientes valores $F$ tabulados con nivel de significancia a del $95 \%$ [13]; por lo tanto, el efecto de la concentración de catalizador, del $\mathrm{pH}$ y de las interacciones entre éstos, no influyen de manera significativa en la eficacia de remoción de la DQO de las aguas empleadas. En la figura 2 se muestran las medias de los porcentajes de remoción de $\mathrm{DQO}$, en función de la relación $\mathrm{Fe}^{2+} / \mathrm{H}_{2} \mathrm{O}_{2}$ (a) y del pH (b). 

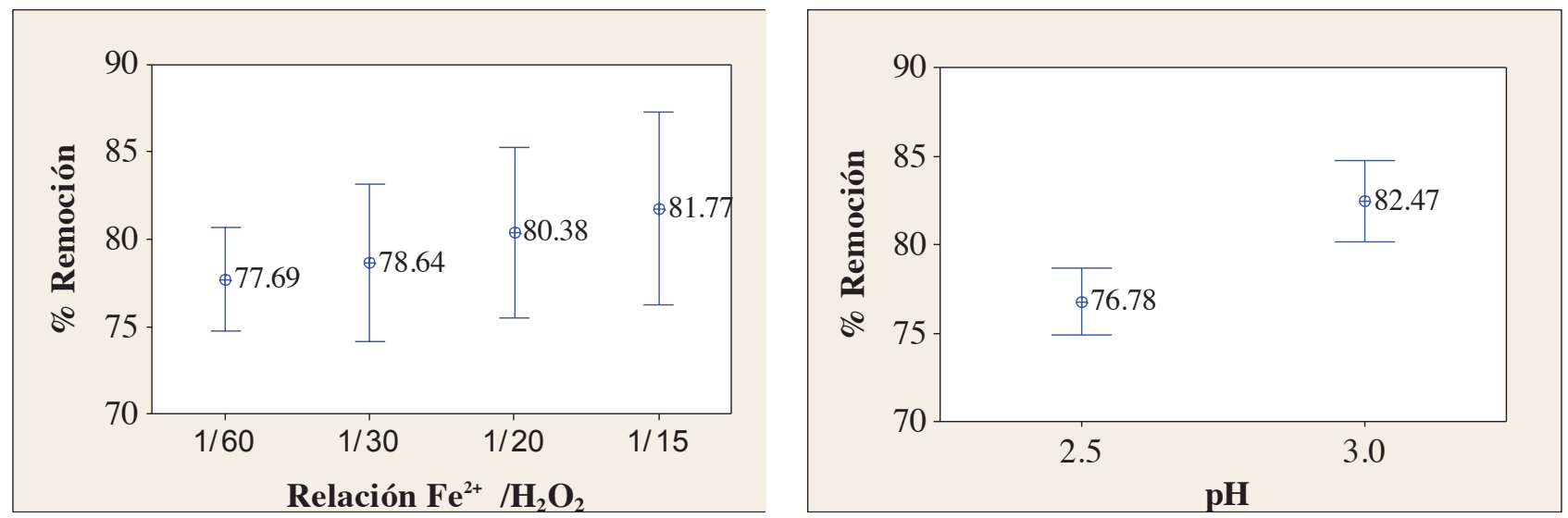

Figura 2. Medias de \% remoción $D Q O$ e intervalos de confianza al 95\%: a) para la relación $\mathrm{Fe}^{2+} / \mathrm{H}_{2} \mathrm{O}_{2}$ y b) para el $\mathrm{pH}$.

En los valores de $\mathrm{pH}$ estudiados, se obtienen porcentajes de remoción de materia orgánica entre 72.51 y $89.68 \%$ de DQO, (según la tabla 2) por lo que el control exhaustivo de este factor no se hace necesario. En la remoción de materia orgánica de aguas residuales de la planta termoeléctrica se pueden manejar valores de $\mathrm{pH}$ cercanos a 3.0, con la dosis de catalizador inferior (relación $\mathrm{Fe}^{2+} / \mathrm{H}_{2} \mathrm{O}_{2}=1 / 60$ ). Varios autores como K. Barbusinski [6] han reportado rangos óptimos de $\mathrm{pH}$ entre 3.0 y 3.5 en la eliminación de pesticidas; Bautista entre 2.5 y 3.0 para reducir contaminantes resultantes de la producción de cosméticos [3]; Blanco alrededor de 2.7 para colorantes textiles [8], de mane- ra que las condiciones óptimas del proceso Fenton, dependen de la naturaleza del agua residual tratada [5].

\subsection{Evolución del proceso Fenton como función del tiempo}

En la Figura 2 se resume la variación de la disminución de materia orgánica respecto del tiempo de contacto, para una muestra con DQO inicial de 357.14 $\mathrm{mgO}_{2} / \mathrm{L}$. Como puede observarse, durante el proceso, existe un descenso brusco en DQO antes de transcurridos los 30 minutos, hasta disminuir paulatinamente a un valor de $36.87 \mathrm{mg} \mathrm{O}_{2} / \mathrm{L}$ correspondiente a un rango de remoción del $89.68 \%$.

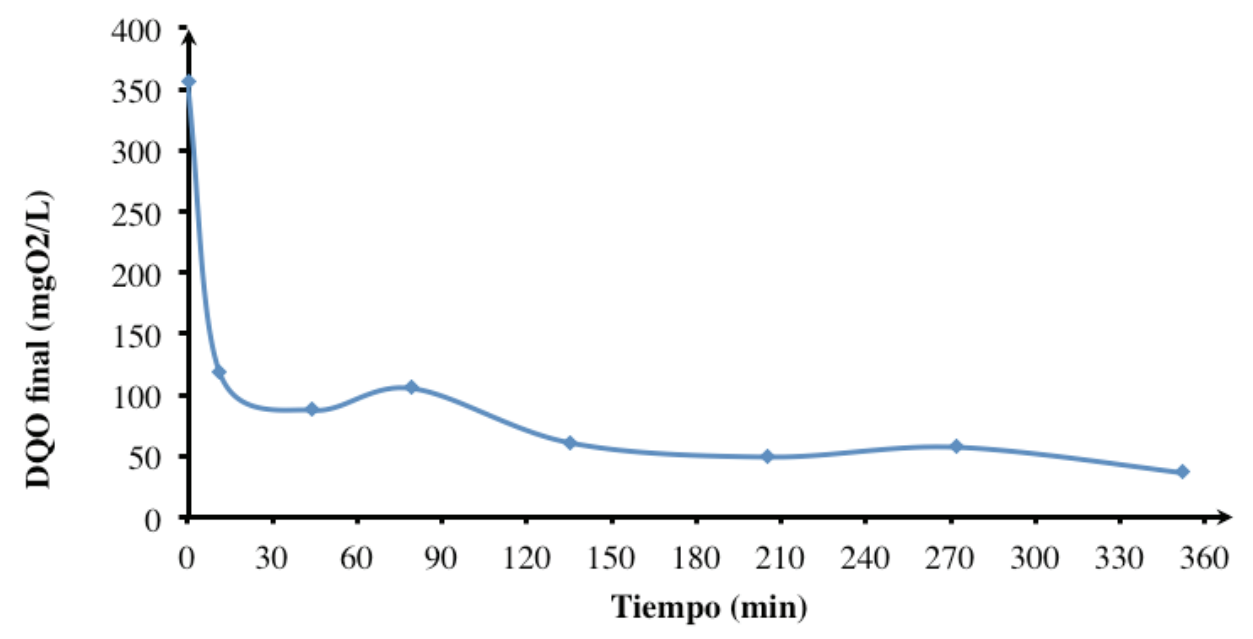

Figura 3. Evolución del proceso Fenton para una muestra de agua residual con DQO inicial de $357.14 \mathrm{mg} \mathrm{O} / \mathrm{L}$ 
Se determinó que 120 minutos fue el mejor tiempo de reacción en el rango estudiado, ya que la remoción promedio se mantiene aproximadamente constante conforme aumenta el tiempo de reacción.

\section{Conclusiones}

El proceso Fenton es eficaz en la remoción de materia orgánica contenida en aguas residuales de la industria termoeléctrica que emplea fuel oil como combustible, ya que logra disminuir significativamente la DQO de estas aguas contaminadas a valores que están muy por debajo del permitido dentro del Distrito Metropolitano de Quito (DMQ). Según la norma técnica refe- rida en la Ordenanza 213, el valor límite es de 123 $\mathrm{mg} \mathrm{O}_{2} / \mathrm{L}$. La aplicación de este proceso de oxidación avanzada no requiere el aporte de energía extra para obtener amplias remociones de carga orgánica en las aguas residuales de estudiadas, pudiéndose trabajar a presión y temperatura ambiente.

\section{Agradecimientos}

Laboratorio de Química Ambiental de la Facultad de Ciencias Químicas de la Universidad Central del Ecuador, Laboratorio Químico y al área de Gestión Ambiental de la Unidad de Negocio Termo-Pichincha de CELEC.

\section{Referencias bibliográficas}

1 Alvarez, C. A., Pérez, C., \& Velando, A. (2007). Effects of acute exposure to heavy fuel oil from the Prestige spill on a seabird. Aquatic Toxicology, 84(1), 103-109.

2 Manahan, S. (2007). Introducción a la Química Ambiental. Barcelona: Reverté.

3 Bautista, P. (2008). Tratamiento de aguas residuales de la industria cosmética mediante el proceso Fenton y con el sistema $\mathrm{Fe} / \mathrm{r}^{-} \mathrm{Al}_{2} \mathrm{O}_{3} /$ $\mathrm{H}_{2} \mathrm{O}_{2}$. Madrid, España. Recuperado el 30 de Marzo de 2009 de http://digitool-uam.greendata.es.

4 Arroyave, J., Garcés, L., \& Cruz, A. (2006). Fotodegradación del Pesticida Mercted Empleando Fotofenton con Lámpara de Luz Ultravioleta. Revista Lasallista de investigación, 3(2), 9-24.

5 Brillas, E., Sirés, I., \& Oturan, M. (2009). Electo-Fenton Process and Related Electrochemical Technologies Based on Fenton`s Reaction Chemistry. Chemical Reviews , 109(12), 6570-6616.

6 Barbusinski, K., \& Filipek, K. (2001). Use of Fenton's Reagent for Removal of Pesticides from Industrial Wastewater. Polish Journal of Environmental Studies, 10(4), 207-211.

7 Méndez, R., Refugio, G., Castillo, E., \& Sauri, M. (2010). Tratamiento de lixiviados por oxidación Fenton. Ingeniería e Investigación, 30(1), 80-85.

8 Blanco, J. (2009). Degradación de un efluente real textil mediante procesos Fenton y Foto-Fenton. Barcelona, España. Recuperado el 11 de abril de 2010 de http://upcommons.upc.edu

9 Beltran de Heredia, J., Torregrosa, J., Dominguez, J., \& Partido, E. (2005). Degradation of wine distillery wastewaters by the combination of aerobic biological treatment with chemical oxidation by Fenton's reagent. Water Science and Technology, 51(1), 167-174.

10 Vergara, J., Silva, S., \& Trejo, M. d. (2008). Degradation of Corn Oil Wastes By Fenton Reaction and Under Mildly Basic Media in the Presence of Oxidants Assisted with sun Light. American Journal of Environmental Sciences , 4(6), 602-607.

11 APHA, AWWA, \& WEF. (2005). Standard Methods for the Examination of Water and Wastewater. Washington D.C.: American Public Health Asociation.

12 Montgomery. (2003). Diseño y análisis de experimentos (Segunda edición.). México: Limusa Wiley.

13 Gutiérrez, H. (2004). Análisis y Diseño de Experimentos. Guadalajara: McGraw-Hill. 